\title{
On the connection coefficients and recurrence relations arising from expansions in series of modified generalized Laguerre polynomials: Applications on a semi-infinite domain
}

https://doi.org/10.1515/nleng-2018-0073

Received April 29, 2018; revised June 3, 2018; accepted July 6, 2018.

Abstract: Herein, three important theorems were stated and proved. The first relates the modified generalized Laguerre expansion coefficients of the derivatives of a function in terms of its original expansion coefficients; and an explicit expression for the derivatives of modified generalized Laguerre polynomials of any degree and for any order as a linear combination of modified generalized Laguerre polynomials themselves is also deduced. The second theorem gives new modified generalized Laguerre coefficients of the moments of one single modified generalized Laguerre polynomials of any degree. Finally, the third theorem expresses explicitly the modified generalized Laguerre coefficients of the moments of a generalorder derivative of an infinitely differentiable function in terms of its modified generalized Laguerre coefficients. Some spectral applications of these theorems for solving ordinary differential equations with varying coefficients and some specific applied differential problems, by reducing them to recurrence relations in their expansion coefficients of the solution are considered.

Keywords: Generalized Laguerre Polynomials; spectral methods; Lane-Emden equation; Bratu's equation; Burger's equation

\footnotetext{
*Corresponding Author: E.H. Doha, Department of Mathematics, Faculty of Science, Cairo University, Giza 12613, Egypt, E-mail: eiddoha@sci.cu.edu.eg, ORCID: http://orcid.org/0000-0002-7781-6871 Y.H. Youssri, Department of Mathematics, Faculty of Science, Cairo University, Giza 12613, Egypt, E-mail: youssri@sci.cu.edu.eg, ORCID: http://orcid.org/0000-0003-0403-8797
}

\section{Introduction}

Classical Laguerre polynomials [1-4] are the main subject of a very extensive literature. If we denote by $\left\{L_{n}^{(\alpha)}(x): n=0,1,2, \cdots\right\}$, the sequence of monic Laguerre polynomials, then their crucial property is the following orthogonality condition

$$
\int_{0}^{\infty} L_{m}^{(\alpha)}(x) L_{n}^{(\alpha)}(x) x^{\alpha} e^{-\chi} d x=\frac{\Gamma(n+\alpha+1)}{n !} \delta_{m n},
$$

where $m, n \in \mathbb{N}$ and the parameter $\alpha$ satisfies the condition $\alpha>-1$ in order to assure the convergence of the integrals. For more details about classical Laguerre polynomials, please see [5].

In this framework, we define the modified generalized Laguerre polynomials $L_{n}^{(\alpha, \beta)}(x)$, with the newly added parameter $\beta$, the existence of this parameter will affect on the convergence of $L_{n}^{(\alpha, \beta)}(x)$, moreover, the classical Laguerre polynomials can be obtained as a direct special case of $L_{n}^{(\alpha, \beta)}(x)$ by letting $\beta=1$.

Many differential models in various research disciplines such as mathematics, fluid dynamics, chemistry, biology, viscoelasticity, engineering and physics have arisen in semi-infinite domains [6-10]. Consequently, a lot of researchers have utilized various transformations on classical orthogonal polynomials to map the bounded interval $[-1,1]$ into $[0, L]$ and $[0, \infty)$ maintaining their orthogonal property. This idea is acceptable, nevertheless, the rate of convergence as well as the stability of solutions will affected when $L \gg 1$ also when the number of retained modes is great. In this research we tame the proposed orthogonal polynomials $L_{n}^{(\alpha, \beta)}(x)$ to handle some differential problems on a semi-infinite domain.

To be more precise we tackle three important problems over a semi-infinite domain, namely, the LaneEmden equation, Bratu's type equation and the nonlinear space-time Burger's type equation. 
The Lane-Emden equation [11-13] is one of the basic equations in the theory of stellar structure and has been the focus of many studies. This equation describes the temperature variation of a spherical gas cloud under the mutual attraction of its molecules and subject to the laws of classical thermodynamics. It also describes the variation of density as a function of the radial distance for a polytrope.

The initial value problems of Bratu's type $[14,15]$ was used to model a combustion problem in a numerical slab. Bratu's problem is also used in a large variety of applications such as the fuel ignition model of the thermal combustion theory, the model of the thermal reaction process, the Chandrasekhar model [16] of the expansion of the universe, questions in geometry and relativity concerning the Chandrasekhar model, chemical reaction theory, radiative heat transfer and nanotechnology.

The one-dimensional Burgers equation first appeared in a paper by Bateman [17], who derived two of the essentially steady solutions. It is a special case of some mathematical models of turbulence introduced about twenty years ago by Burgers [18]. The distinctive feature of Burger's equation is that it is the simplest mathematical formulation of the competition between convection and diffusion. It thus offers a relatively convenient means of studying not only turbulence but also the distortion caused by laminar transport of momentum in an otherwise symmetric disturbance and the decay of dissipation layers formed.

In this work, we attempt to solve differntial equations using the modified generalized Laguerre polynomials jointly with the spectral accurate tau and collocation methods. The Laguerre polynomials and the spectral collocation method for the numerical solution of differential equations have been extensively used in [19-24]. Guo et al. [25] applied the mixed generalized Laguerre spectral method for the exterior problems. In [26], the mixed Laguerre-Legendre spectral method and pseudospectral method are proposed for solving the fluid folw problems in an infinite channel. Rahmoune [27], presented a spectral collocation method using the scaled Laguerre functions for solving the Fredholm integral equations of the second kind on the half-line. In [28], a spectral collocation method based on the Laguerre polynomials has been proposed for solving the fractional pantograph equation.

In most of these applications, a formula is used that relates the expansion coefficients of derivatives appearing in the differential equation to those of the function itself. For the Galerkin and tau variants of the spectral methods, explicit expressions for the expansion coefficients for the solution are needed. Karageorghis [29], obtained an expression when the basis functions of expansion are shifted Chebyshev polynomials $T_{n}^{\star}(x), x \in[0,1]$. A corresponding formula for Legendre polynomials $P_{n}(x), x \in[-1,1]$, is derived by Phillips [30]. Doha [31], has obtained a more general formula when the basis functions are the ultraspherical polynomials $C_{n}^{(\lambda)}(x), x \in[-1,1], \lambda \in\left(-\frac{1}{2}, \infty\right)$; formulae for the first and second kinds of Chebyshev polynomials and Legendre polynomials $T_{n}(x), U_{n}(x)$ and $P_{n}(x)$ are given as special cases of $C_{n}^{(\lambda)}(x)$. A most general formula when the basis functions are the Jacobi polynomials $P_{n}^{(v, \mu)}(x), x \in[-1,1], v>-1, \mu>-1$, is given in Doha [32]. Another formula when the basis functions are the Hermite polynomials is obtained in Doha [33], two other formulae for the third and fourth kinds of Chebyshev polynomials $V_{n}(x)$ and $W_{n}(x)$ are given in Doha and Abd-Elhameed [34].

A more general situation which often arises in the numerical solution of differential equations with polynomial coefficients in spectral methods is the evaluation of the expansion coefficients of the moments of high-order derivatives of infinitely differentiable functions. A formula for the shifted Chebyshev coefficients of the moments of generalorder derivatives of an infinitely differentiable function is given in Karageorghis [29]. Corresponding results for Chebyshev polynomials of the first and second kinds, Legendre, ultraspherical and Hermite polynomials are given in Doha [35], Doha and El-Soubhy [36], Doha [37] and Doha [38], respectively, another work on connection formulae were given in [39-41].

Up to now, and to the best of our knowledge, many formulae corresponding to those mentioned previously are not known and traceless in the literature for the modified generalized Laguerre expansions. This motivates our interest in such polynomials. Another motivation is that the theoretical and numerical analyses of numerous physical and mathematical problems very often require the expansion of an arbitrary polynomial or the expansion of an arbitrary function with its derivatives and moments into a set of orthogonal polynomials. This is in particular true (for modified generalized Laguerre polynomials) in quantum mechanical studies of physical systems, where the equation of motion or Schrpdinger equation is a secondorder differential equation with polynomial coefficients. This is the case not only for the solution of the Schrödinger, Klein-Gordon and Dirac equations for the Coulomb field but also for many other potentials, as shown for example in Bagrov and Gitman [42] and Nikiforov and Uvarov [43]. 
The presentation of the paper is as follows, in Section 2, we present some important properties of the modified generalized Laguerre polynomials. In Section 3, we state and prove relations between the coefficients $a_{n}^{(q)}$ and $a_{n}$ and the $q$ th derivative of $L_{n}^{(\alpha, \beta)}(x)$. In Section 4, we present explicit relation for the modified generalized Laguerre coefficients of the moments of one single modified Laguerre polynomial of any degree. In Section 5 , we present the modified generalized Laguerre coefficients of a generalorder derivative of an infinitely differentiable function in terms of its modified Laguerre coefficients. In Section 6, we implement an application to ordinary differential equations with variable coefficients. Finally, in Section 7, we present four applications for solving second-order differential equations with variable coefficients, namely, the Lane-Emden equation, Bratu's type equation and the nonlinear space-time Burger’s type equation.

\section{Some properties of modified generalized Laguerre polynomials}

Let $w_{\alpha, \beta}(x)=x^{\alpha} e^{-\beta x}$ with $\alpha>-1$ and $\beta>0$. Then the modified generalized Laguerre polynomials (MGLP) are a sequence of polynomials $\left\{L_{n}^{(\alpha, \beta)}(x): n=0,1,2, \cdots\right\}$, each of degree $n$, satisfying the orthogonality relation

$$
\begin{gathered}
\int_{0}^{\infty} w_{\alpha, \beta}(x) L_{m}^{(\alpha, \beta)}(x) L_{n}^{(\alpha, \beta)}(x) d x=\frac{\Gamma(n+\alpha+1)}{n ! \beta^{\alpha+1}} \delta_{m n}, \\
\alpha>-1, \beta>0,
\end{gathered}
$$

where $\delta_{m n}$ is the kronecker symbol. The set of (MGLP) is a complete $L_{w_{\alpha, \beta}(x)}^{2}(0, \infty)$-orthogonal system. The modified generalized Laguerre polynomials may be generated by using Rodrigue's formula

$$
L_{n}^{(\alpha, \beta)}(x)=\frac{1}{n ! w_{\alpha, \beta}(x)} \frac{d^{n}}{d x^{n}}\left[x^{n+\alpha} e^{-\beta x}\right], n=0,1,2, \cdots .
$$

The following two recurrence relations are of fundamental importance in developing the present work. These are

$$
\begin{aligned}
(n+1) L_{n+1}^{(\alpha, \beta)}(x)= & (2 n+\alpha+1-\beta x) L_{n}^{(\alpha, \beta)}(x) \\
& -(n+\alpha) L_{n-1}^{(\alpha, \beta)}(x), n=0,1,2, \cdots,
\end{aligned}
$$

with $L_{-1}^{(\alpha, \beta)}(x)=0$, and

$$
L_{n}^{(\alpha, \beta)}(x)=\frac{1}{\beta} D\left[L_{n}^{(\alpha, \beta)}(x)-L_{n+1}^{(\alpha, \beta)}(x)\right], n=0,1,2, \cdots,
$$

where $D \equiv \frac{d}{d x}$. Note that the recurrence relation (4) may be used to generate the (MGLP) starting from $L_{0}^{(\alpha, \beta)}(x)=1$, and $L_{1}^{(\alpha, \beta)}(x)=1+\alpha-\beta x$. If $\beta=1$, then $L_{n}^{(\alpha, \beta)}(x)$ is the standard modified generalized Laguerre polynomials.

Suppose now we are given a function $f(x)$ which is infinitely differentiable in the interval $[0, \infty)$, then we can write

$$
f(x)=\sum_{n=0}^{\infty} a_{n} L_{n}^{(\alpha, \beta)}(x),
$$

and for the $q$ th derivative of $f(x)$,

$$
f^{(q)}(x)=\sum_{n=0}^{\infty} a_{n}^{(q)} L_{n}^{(\alpha, \beta)}(x), \quad a_{n}^{(0)}=a_{n} .
$$

Moreover, if $f(x)$ satisfies, $f(x)=O\left(e^{\alpha x}\right)$ for $x \rightarrow \infty$, and for some $\alpha<\frac{1}{2}$, then it can be shown (cf, [44]) that the modified generalized Laguerre expansion

$$
f(x)=\sum_{n=0}^{N} a_{n} L_{n}^{(\alpha, \beta)},
$$

converges faster than algebraically as the number of terms $N \rightarrow \infty$

\section{Relations between the coefficients $a_{n}^{(q)}$ and $a_{n}$ and the $q$ th derivative of $L_{n}^{(\alpha, \beta)}(x)$}

Theorem 1. If $f(x)$ is infinitely differentiable and expanded as in (5), and the qth derivative of $f(x)$ is expressed as in (6), then

$$
a_{n}^{(q)}=\beta^{q} \sum_{j=0}^{n}\left(\begin{array}{c}
n-j+q-1 \\
q-1
\end{array}\right) a_{j}, q \geq 0,
$$

and

$$
D^{q} L_{n}^{(\alpha, \beta)}(x)=(-\beta)^{q} \sum_{j=0}^{n-q}\left(\begin{array}{c}
n-j-1 \\
q-1
\end{array}\right) L_{j}^{(\alpha, \beta)}(x) .
$$

Proof. It is clear that

$$
f^{(q+1)}(x)=\sum_{n=0}^{\infty} a_{n}^{(q+1)} L_{n}^{(\alpha, \beta)}(x)
$$

and on differentiating (6), and making use of (4), we get

$$
a_{n}^{(q+1)}-a_{n-1}^{(q+1)}=\beta a_{n}^{(q)}, n=0,1,2, \cdots, q \geq 0
$$


which immediately gives

$$
a_{n}^{(q+1)}=\beta \sum_{j=0}^{n} a_{j}^{(q)}
$$

and this in turn yields

$$
\begin{aligned}
& a_{n}^{(1)}=\beta \sum_{j=0}^{n} a_{j}, \\
& a_{n}^{(2)}=\beta^{2} \sum_{j=0}^{n}(n-j+1) a_{j}, \\
& a_{n}^{(3)}=\beta^{3} \sum_{j=0}^{n} \frac{(n-j+1)(n-j+2)}{2} a_{j},
\end{aligned}
$$

and finally

$$
a_{n}^{(q)}=\beta^{q} \sum_{j=0}^{n} \frac{(n-j+1)(n-j+2) \cdots(n-j+q-1)}{q-1} a_{j},
$$

i.e.

$$
a_{n}^{(q)}=\beta^{q} \sum_{j=0}^{n}\left(\begin{array}{c}
n-j+q-1 \\
q-1
\end{array}\right) a_{j},
$$

which proves (7). From the properties of modified generalized Laguerre polynomials, it can be easily shown that,

$$
D L_{n}^{(\alpha, \beta)}(x)=-\beta \sum_{j=0}^{n-1} L_{j}^{(\alpha, \beta)}(x),
$$

which immediately gives

$$
D^{q} L_{n}^{(\alpha, \beta)}(x)=(-\beta)^{q} \sum_{j=0}^{n-q}\left(\begin{array}{c}
n-j-1 \\
q-1
\end{array}\right) L_{j}^{(\alpha, \beta)}(x)
$$

and this completes the proof of Theorem 1 .

\section{Modified generalized Laguerre coefficients of the moments of one single (MGLP) of any degree}

For the evaluation of modified generalized Laguerre coefficients of the moments of higher-order derivatives of infinitely differentiable functions, the following theorem is needed.

\section{Theorem 2.}

$$
\beta^{m} x^{m} L_{j}^{(\alpha, \beta)}(x)=\sum_{n=0}^{2 m} a_{m n}(j) L_{j+m-n}^{(\alpha, \beta)}(x), m \geq 0, j \geq 0,
$$

where

$$
\begin{aligned}
a_{m n}(j)= & \frac{(-1)^{m-n}(m !)^{2}}{\Gamma(j+m-n+\alpha+1)} \sum_{k=\max (0, j-n)}^{\min (j+m-n, j)}\left(\begin{array}{c}
j+m-n \\
k
\end{array}\right) \\
& \frac{\Gamma(m+k+\alpha+1)}{(j-k) !(n-j+k) !(m-j+k) !}
\end{aligned}
$$

Proof. We use the induction principle to prove this theorem. In view of the recurrence relation (3), we may write

$$
\begin{aligned}
\beta x L_{j}^{(\alpha, \beta)}(x)= & -(j+1) L_{j+1}^{(\alpha, \beta)}(x)+(2 j+\alpha+1) L_{j}^{(\alpha, \beta)}(x) \\
& -(j+\alpha) L_{j-1}^{(\alpha, \beta)}(x),
\end{aligned}
$$

which may be put in the form

$$
\begin{aligned}
\beta x L_{j}^{(\alpha, \beta)}(x)= & a_{10}(j) L_{j+1}^{(\alpha, \beta)}(x)+a_{11}(j) L_{j}^{(\alpha, \beta)}(x) \\
& +a_{12}(j) L_{j-1}^{(\alpha, \beta)}(x),
\end{aligned}
$$

this in turn shows that (9) is true for $m=1$. Proceeding by induction, assuming that (9) is valid for $m$, we want to prove that

$$
\beta^{m+1} x^{m+1} L_{j}^{(\alpha, \beta)}(x)=\sum_{n=0}^{2 m+2} a_{m+1, n}(j) L_{j+m-n+1}^{(\alpha, \beta)}(x) .
$$

From (11) and assuming the validity of (9), we have

$$
\begin{aligned}
& \beta^{m+1} x^{m+1} L_{j}^{(\alpha, \beta)}(x)=\sum_{n=0}^{2 m} a_{m, n}(j)\left[a_{10}(j+m-n) L_{j+m-n+1}^{(\alpha, \beta)}(x)\right. \\
& \left.+a_{11}(j+m-n) L_{j+m-n}^{(\alpha, \beta)}(x)+a_{12}(j+m-n) L_{j+m-n-1}^{(\alpha, \beta)}(x)\right] .
\end{aligned}
$$

Collecting similar terms, we get

$\beta^{m+1} x^{m+1} L_{j}^{(\alpha, \beta)}(x)=a_{m 0}(j) a_{10}(j+m) L_{j+m+1}^{(\alpha, \beta)}(x)$

$+\left[a_{m 1}(j) a_{10}(j+m-1)+a_{m 0}(j) a_{11}(j+m)\right] L_{j+m}^{(\alpha, \beta)}(x)$

$+\sum_{n=0}^{2 m}\left[a_{m, n}(j) a_{10}(j+m-n)+a_{m, n-1}(j) a_{11}(j+m-n+1)\right.$

$\left.+a_{m, n-2}(j) a_{12}(j+m-n+2)\right] L_{j+m-n+1}^{(\alpha, \beta)}(x)$

$+\left[a_{m, 2 m}(j) a_{11}(j-m)+a_{m, 2 m-1}(j) a_{12}(j-m+1)\right] L_{j-m}^{(\alpha, \beta)}(x)$

$+a_{m, 2 m}(j) a_{12}(j-m) L_{j-m-1}^{(\alpha, \beta)}(x)$.

It can be easily shown that

$$
\begin{aligned}
a_{m+1,0}(j)= & a_{m 0}(j) a_{10}(j+m) \\
a_{m+1,1}(j)= & a_{m 1}(j) a_{10}(j+m-1)+a_{m 0}(j) a_{10}(j+m) \\
a_{m+1, n}(j)= & a_{m n}(j) a_{10}(j+m-n) \\
& +a_{m, n-1}(j) a_{11}(j+m-n+1) \\
& +a_{m, n-2}(j) a_{12}(j+m-n+2) \\
a_{m+1,2 m+1}(j)= & a_{m, 2 m}(j) a_{11}(j-m)+a_{m, 2 m-1}(j) a_{12}(j-m+1) \\
a_{m+1,2 m+2}(j)= & a_{m, 2 m}(j) a_{12}(j-m)
\end{aligned}
$$


and accordingly, formula (14) becomes,

$$
\beta^{m+1} x^{m+1} L_{j}^{(\alpha, \beta)}(x)=\sum_{n=0}^{2 m+2} a_{m+1, n}(j) L_{j+m-n+1}^{(\alpha, \beta)}(x),
$$

which completes the induction and proves the theorem.

It is worth noting here that, recalling the definition of Pochhammer's symbol,

$$
(z)_{n}=\frac{\Gamma(z+n)}{\Gamma(z)}=\frac{(-1)^{n} \Gamma(1-z)}{\Gamma(1-z-n)},
$$

and the identity

$$
\left(\begin{array}{l}
n \\
k
\end{array}\right)=\frac{(-1)^{k}(-n)_{k}}{k !},
$$

formula (10) can be written in terms of $\mathrm{a}_{3} F_{2}$ hypergeometric function of unit argument.

$$
\begin{aligned}
& a_{m n}(j)=\frac{(-1)^{m-n}(m !)^{2} \Gamma(m+\alpha+1)}{j !(n-j) !(m-j) ! \Gamma(j+m-n+\alpha+1)}
\end{aligned}
$$

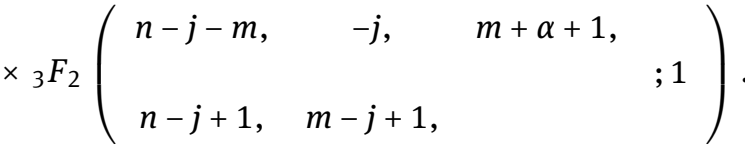

Corollary 1. It is not difficult to show that

$$
\beta^{m} x^{m} L_{j}^{(\alpha, \beta)}(x)=\sum_{n=0}^{j+m} a_{m, j+m-n}(j) L_{n}^{(\alpha, \beta)}(x), \quad j \geq 0, m \geq 0
$$

and

$$
\beta^{m} x^{m}=\sum_{n=0}^{m} a_{m, m-n}(0) L_{n}^{(\alpha, \beta)}(x), \quad m \geq 0
$$

where

$$
a_{m, m-n}(0)=\frac{(-1)^{n} m ! \Gamma(m+\alpha+1)}{(m-n) ! \Gamma(n+\alpha+1)} .
$$

\section{Modified generalized Laguerre coefficients of a general-order derivative of an infinitely differentiable function}

Theorem 3. Let $f(x)$ and all its derivatives be smooth and $f(x)$ and $f^{(q)}(x)$ be expanded as in (5) and (6), respectively, and for a positive integral $\ell$, let

$$
\beta^{\ell} \chi^{\ell} f^{(q)}(x)=I^{q, \ell},
$$

and if we write

$$
I^{q, \ell}=\sum_{j=0}^{\infty} b_{i}^{q, \ell} L_{i}^{(\alpha, \beta)}(x),
$$

then

$$
b_{i}^{q, \ell}=\left\{\begin{array}{l}
\sum_{k=0}^{\ell-1} a_{\ell, k+\ell-i}(k) a_{k}^{(q)}+\sum_{k=0}^{i} a_{\ell, k+2 \ell-i}(k+\ell) a_{k+\ell}^{(q)}, \\
0 \leq i \leq \ell ; \\
\sum_{k=i-\ell}^{\ell-1} a_{\ell, k+\ell-i}(k) a_{k}^{(q)}+\sum_{k=0}^{i} a_{\ell, k+2 \ell-i}(k+\ell) a_{k+\ell}^{(q)}, \\
\ell+1 \leq i \leq 2 \ell-1 ; \\
\sum_{k=i-2 \ell}^{i} a_{\ell, k+2 \ell-i}(k+\ell) a_{k}^{(q)}, \\
i \geq 2 \ell .
\end{array}\right.
$$

Proof. Relations (6), (9) and (17) give

$$
I^{q, \ell}=\sum_{k=0}^{\infty} a_{k}^{(q)} \sum_{j=0}^{2 \ell} a_{\ell, j}(k) L_{k+\ell-j}^{(\alpha, \beta)}(x) .
$$

By letting $i=k+\ell-j$, (20) may be written in the form

$$
\begin{aligned}
I^{q, \ell}= & \sum_{k=0}^{\ell-1} a_{k}^{(q)} \sum_{i=k-\ell}^{k+\ell} a_{\ell, k+\ell-i}(k) L_{i}^{(\alpha, \beta)}(x) \\
& +\sum_{k=\ell}^{\infty} a_{k}^{(q)} \sum_{i=k-\ell}^{k+\ell} a_{\ell, k+\ell-i}(k) L_{i}^{(\alpha, \beta)}(x)=\sum_{1}+\sum_{2},
\end{aligned}
$$

where

$$
\begin{aligned}
& \sum_{1}=\sum_{k=0}^{\ell-1} a_{k}^{(q)} \sum_{i=k-\ell}^{k+\ell} a_{\ell, k+\ell-i}(k) L_{i}^{(\alpha, \beta)}(x), \\
& \sum_{2}=\sum_{k=\ell}^{\infty} a_{k}^{(q)} \sum_{i=k-\ell}^{k+\ell} a_{\ell, k+\ell-i}(k) L_{i}^{(\alpha, \beta)}(x) .
\end{aligned}
$$

Considering $\sum_{1}$ first,

$$
\begin{aligned}
\sum_{1}= & \sum_{k=0}^{\ell-1} a_{k}^{(q)} \sum_{i=k-\ell}^{-1} a_{\ell, k+\ell-i}(k) L_{i}^{(\alpha, \beta)}(x) \\
& +\sum_{k=0}^{\ell-1} a_{k}^{(q)} \sum_{i=0}^{k+\ell} a_{\ell, k+\ell-i}(k) L_{i}^{(\alpha, \beta)}(x)=\sum_{11}+\sum_{12} .
\end{aligned}
$$

Clearly,

$$
\begin{aligned}
\sum_{11} & =\sum_{k=0}^{\ell-1} a_{k}^{(q)} \sum_{i=k-\ell}^{-1} a_{\ell, k+\ell-i}(k) L_{i}^{(\alpha, \beta)}(x) \\
& =\sum_{k=0}^{\ell-1} a_{k}^{(q)} \sum_{i=1}^{\ell-k} a_{\ell, k+\ell+i}(k) L_{-i}^{(\alpha, \beta)}(x)
\end{aligned}
$$


hence

$$
\begin{gathered}
\sum_{11}=0 . \\
\sum_{12}=\sum_{k=0}^{\ell-1} a_{k}^{(q)} \sum_{i=0}^{k+\ell} a_{\ell, k+\ell-i}(k) L_{i}^{(\alpha, \beta)}(x) \\
=\sum_{i=0}^{\ell} \sum_{k=0}^{\ell-1} a_{k}^{(q)} a_{\ell, k+\ell-i}(k) L_{i}^{(\alpha, \beta)}(x) \\
+\sum_{i=\ell+1}^{2 \ell-1} \sum_{k=i-\ell}^{\ell-1} a_{k}^{(q)} a_{\ell, k+\ell-i}(k) L_{i}^{(\alpha, \beta)}(x),
\end{gathered}
$$

hence,

$$
\sum_{12}=\sum_{i=0}^{2 \ell-1} \sum_{k=\max (0, i-\ell)}^{\ell-1} a_{k}^{(q)} a_{\ell, k+\ell-i}(k) L_{i}^{(\alpha, \beta)}(x) .
$$

Substitution of (24) and (25) into (23) yields,

$$
\sum_{1}=\sum_{i=0}^{2 \ell-1} \sum_{k=\max (0, i-\ell)}^{\ell-1} a_{k}^{(q)} a_{\ell, k+\ell-i}(k) L_{i}^{(\alpha, \beta)}(x) .
$$

When considering $\sum_{2}$, if one takes $k+\ell$ instead of $k$, then it is not difficult to show that

$$
\sum_{2}=\sum_{i=0}^{\infty} \sum_{k=\max (0, i-2 \ell)}^{i} a_{k+\ell}^{(q)} a_{\ell, k+2 \ell-i}(k+\ell) L_{i}^{(\alpha, \beta)}(x) .
$$

Substitution of (26) and (27) into (21) gives the required results (19) and completes the proof of Theorem 3.

\section{Application to ordinary differential equations with variable coefficients}

(i) Let $y(x)$ be infinitely differentiable function defined on $[0, \infty)$ and having the Laguerre expansion as in (5), and assume that it satisfies the linear nonhomogenous differential equation of order $n>0$,

$$
\sum_{i=0}^{n} p_{i}(x) y^{(i)}(x)=f(x), \quad x \in[0, \infty),
$$

where $p_{0}, p_{1}, \cdots, p_{n} \neq 0$ are polynomials of $x$, and the coefficients of Laguerre series of $f(x)$ are known; formula (7), (9) and (19) enable one to construct, in view of (28), the linear recurrence relation of order $r$, namely,

$$
\sum_{j=0}^{r} \alpha_{j}(k) a_{k+j}=\beta(k), \quad k \geq 0
$$

where $\alpha_{0}, \alpha_{1}, \cdots, \alpha_{r}\left(\alpha_{0} \alpha_{r} \neq 0\right)$ are polynomials of the variable $k$. The interested reader is referred to [45] for a similar derivation of (29) when the basis of expansion is Jacobi polynomials.

(ii) Consider the linear ordinary differential equation of order $n$ of the form

$$
\sum_{i=0}^{n} f_{i}(x) y^{(i)}(x)=g(x), \quad x \in[0, \infty),
$$

where $f_{i}(x)$ and $g(x)$ are functions of $x$ only. Suppose the equation to be solved in the interval $[0, \infty)$ subject to $n$ linear boundary conditions, and assume we approximate $y(x)$ a truncated expansion of Laguerre polynomials

$$
\sum_{i=0}^{n}\left[f_{i}(x) \sum_{j=0}^{N} a_{j} D^{j} L_{j}^{(\alpha, \beta)}(x)\right]=g(x)
$$

which may be written in the form

$$
\sum_{j=0}^{N}\left[a_{j} \sum_{i=0}^{n} f_{i}(x) a_{j} D^{j} L_{j}^{(\alpha, \beta)}(x)\right]=g(x) .
$$

The boundary conditions associated with (30) give rise to $n$ equations connecting the coefficients $a_{j}$, and the remaining equations may be obtained in two ways:

(1) We may equate the coefficients of the various $L_{i}^{(\alpha, \beta)}(x)$ after expanding the two sides of (33) in Laguerre series.

(2) We may collocate at $m=N-n$ selected points in $(0, \infty)$. The system of equations obtained from the collocation is of the form

$\sum_{j=0}^{N}\left[a_{j} \sum_{i=0}^{n} f_{i}\left(x_{k}\right) a_{j} D^{j} L_{j}^{(\alpha, \beta)}\left(x_{k}\right)\right]=g\left(x_{k}\right), \quad k=1,2, \cdots, m$,

where $x_{k}$ are the collocation points, which are usually chosen at the zeros of $L_{m}^{(\alpha, \beta)}(x)$, (see, for instance, [46]). Since the derivatives $D^{j} L_{j}^{(\alpha, \beta)}(x)$ are now expressible explicitly in terms of $L_{j}^{(\alpha, \beta)}(x)$, then the problem of computing them solved by using the formula (8). Therefore, the resulting linear system obtained from (31) and the $n$ linear boundary conditions can easily be solved using the standard direct solvers.

\section{Applications and Numerical Results}

Example 1. [38] An example dealing with nonhomogenous differential equation with polynomial coefficients is considered in order to clarify application of the results obtained. 
Consider the nonhomogenous differential equation

$$
\begin{aligned}
& 2 x y^{\prime \prime}(x)+(1+4 x) y^{\prime}(x)+(1+2 x) y(x)=e^{-x} \\
& y(0)=0, y^{\prime}(0)=1 .
\end{aligned}
$$

If $e^{-x}$ is expanded in the form

$$
e^{-\chi}=\sum_{i=0}^{\infty} f_{i} L_{i}^{(\alpha, \beta)}(x),
$$

then

$$
f_{i}=\frac{\beta^{\alpha+1}}{(1+\beta)^{i+\alpha+1}}
$$

and if $y(x)$ is expanded in the form

$$
y(x)=\sum_{i=0}^{\infty} a_{i} L_{i}^{(\alpha, \beta)}(x),
$$

then by virtue of formulae (18) and (19), Eq. (34), takes the form

$$
2 b_{i}^{2,1}+b_{i}^{1,0}+4 b_{i}^{2,1}+b_{i}^{0,0}+2 b_{i}^{0,1}=f_{i}, \quad i \geq 0,
$$

where $b_{i}^{q, \ell}$ are as given in (19). Following the procedure of Doha [38], and after some rather lengthy manipulations, we get,

$$
a_{i}=\frac{\beta^{1+\alpha}(1+\alpha-i)}{(1+\beta)^{i+\alpha+2}} .
$$

It is worthy to mention here that, if $\beta=1$, formulae (36) and (39) of $f_{i}$ and $a_{i}$ will reduced to

$$
f_{i}=\frac{1}{2^{i+\alpha+1}}, \quad a_{i}=\frac{(1+\alpha-i)}{2^{i+\alpha+2}},
$$

which is in complete agreement of Doha [38].

\section{Example 2. Consider the Lane-Emden type equation}

$$
x y^{\prime \prime}(x)+2 y^{\prime}(x)+x y(x)=0, \quad y(0)=1, y^{\prime}(0)=0 \text {. }
$$

with the exact solution

$$
y(x)=\frac{\sin x}{x} .
$$

If $y(x)$ is expanded in the form

$$
y(x)=\sum_{i=0}^{N} a_{i} L_{i}^{(\alpha, \beta)}(x),
$$

then by virtue of formulae (18) and (19), Eq. (40), takes the form

$$
b_{i}^{2,1}+2 b_{i}^{1,0}+b_{i}^{0,1}=0, \quad i \geq 0,
$$

where $b_{i}^{q, \ell}$ are as given in (19). In Table 1, we list the maximum absolute error of Example 2, for different values of $\alpha, \beta$ and $N$ over the interval $(0,10)$. In Figures 1,2 , we depict the graph of approximate and absolute error of Example 2, respectively, for the case $\alpha=1, \beta=\frac{1}{2}$ and $N=40$. In Figure 3, we show the convergence of the method when $N$ increases.
Table 1: Maximum absolute errors for Example 2

\begin{tabular}{c|cccc}
\hline$N$ & $\alpha=1, \beta=2$ & $\alpha=1, \beta=1$ & $\alpha=1, \beta=\frac{1}{2}$ & $\alpha=1, \beta=\frac{1}{4}$ \\
\hline 10 & $9.21 .10^{-1}$ & $5.36 .10^{-2}$ & $5.36 .10^{-3}$ & $1.21 .10^{-3}$ \\
20 & $2.59 .10^{-2}$ & $3.47 .10^{-3}$ & $4.25 .10^{-4}$ & $8.37 .10^{-5}$ \\
30 & $9.37 .10^{-2}$ & $6.92 .10^{-4}$ & $8.34 .10^{-5}$ & $2.22 .10^{-5}$ \\
40 & $1.29 .10^{-3}$ & $8.61 .10^{-4}$ & $2.83 .10^{-6}$ & $4.27 .10^{-6}$ \\
\hline
\end{tabular}

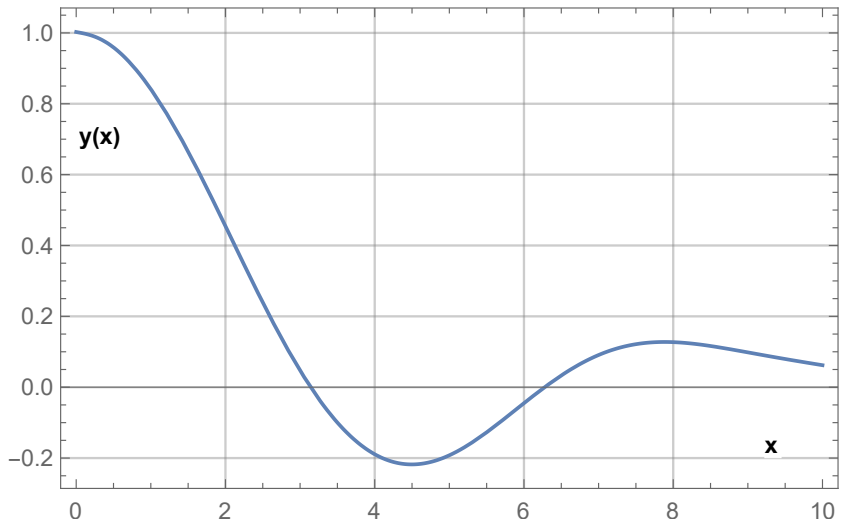

Fig. 1: Approximate solution of Example 2

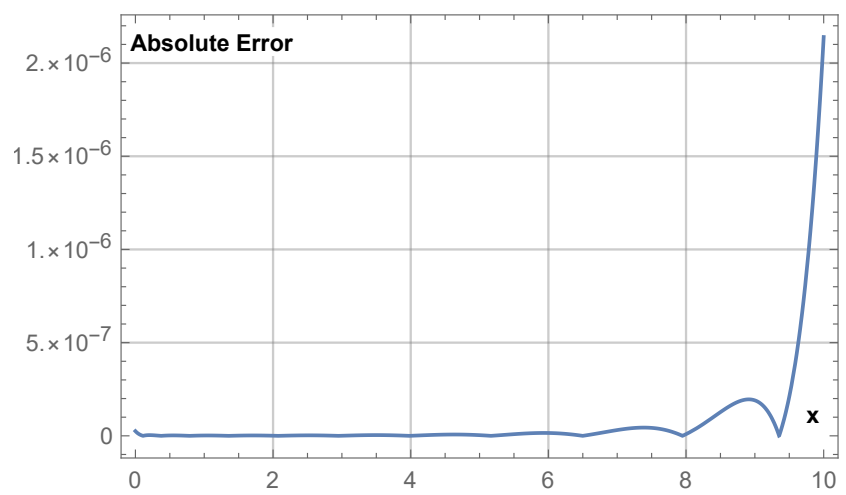

Fig. 2: Absolute Error of Example 2

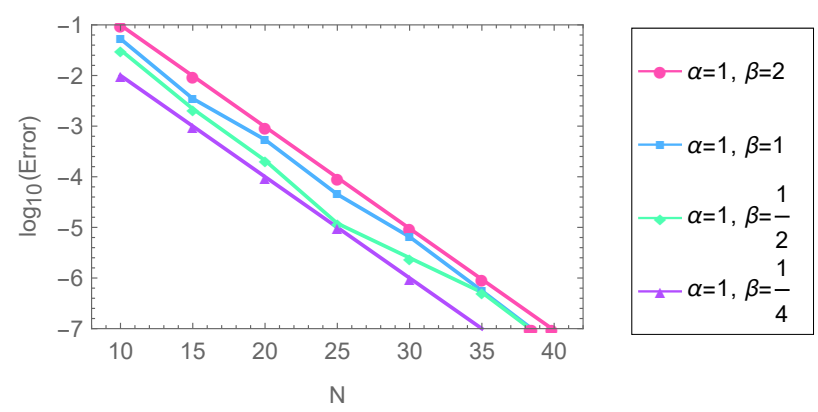

Fig. 3: Log-Error of Example 2

Example 3. Consider the Bratu type equation

$$
y^{\prime \prime}(x)-2 e^{y(x)}=0, \quad y(0)=y^{\prime}(0)=0 .
$$


with the exact solution

$$
y(x)=-\log \left(\cos ^{2} x\right) .
$$

If $y(x)$ is expanded in the form

$$
y(x)=\sum_{i=0}^{N} a_{i} L_{i}^{(\alpha, \beta)}\left(x_{k}\right),
$$

here, we apply the collocation technique. In Table 2, we list the maximum absolute error of Example 3, for different values $\alpha, \beta$ and $N$ over the interval $(0,1.57)$. In Figures 4 and 5 , we depict the graph of approximate and absolute error of Example 3, respectively, for the case $\alpha=1, \beta=\frac{1}{4}$ and $N=20$. In Figure 6, we show the convergence of the method when $N$ increases.

Table 2: Maximum absolute errors for Example 3

\begin{tabular}{c|cccc}
\hline$N$ & $\alpha=1, \beta=2$ & $\alpha=1, \beta=1$ & $\alpha=1, \beta=\frac{1}{2}$ & $\alpha=1, \beta=\frac{1}{4}$ \\
\hline 5 & $8.12 .10^{-1}$ & $2.27 .10^{-2}$ & $6.29 .10^{-2}$ & $3.27 .10^{-2}$ \\
10 & $9.76 .10^{-2}$ & $3.86 .10^{-3}$ & $2.67 .10^{-3}$ & $4.44 .10^{-3}$ \\
15 & $7.38 .10^{-2}$ & $4.97 .10^{-4}$ & $2.81 .10^{-3}$ & $3.67 .10^{-4}$ \\
20 & $5.38 .10^{-3}$ & $9.37 .10^{-4}$ & $3.94 .10^{-4}$ & $1.00 .10^{-5}$ \\
\hline
\end{tabular}

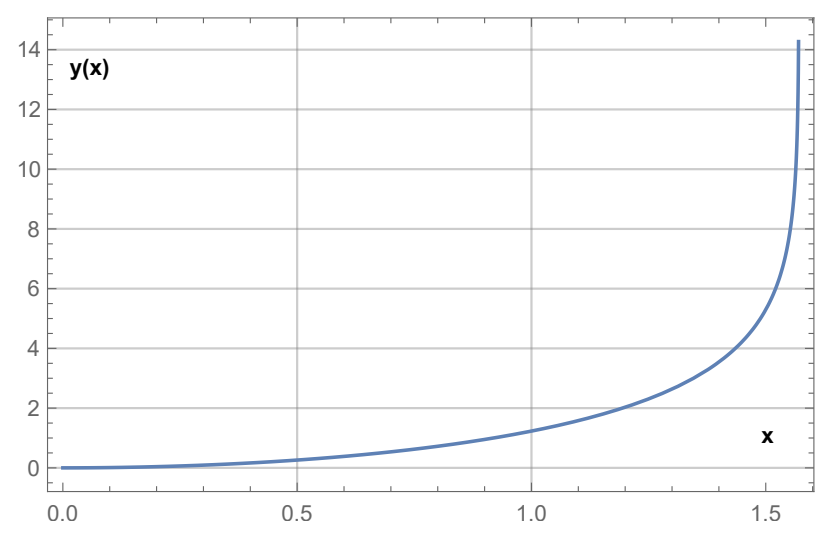

Fig. 4: Approximate solution of Example 3

Example 4. Consider the space-time nonlinear Burgers type equation

$$
\begin{gathered}
u_{t}(x, t)-\mu u_{x x}(x, t)+u(x, t) u_{x}(x, t)=\frac{\pi}{2} e^{-2 \pi^{2} \mu t} \sin (2 \pi x), \\
(x, t) \in(-1,1) \times(0, \tau)
\end{gathered}
$$

subject to the homogenous boundary conditions

$$
u( \pm 1, t)=0,
$$

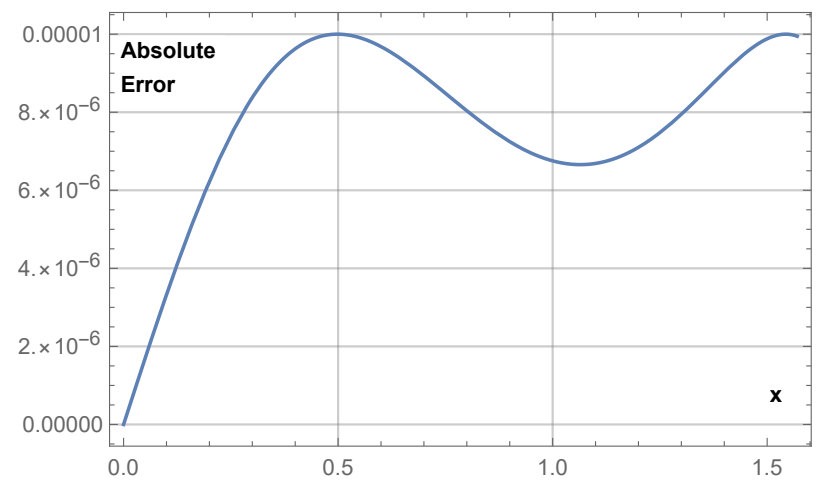

Fig. 5: Absolute Error of Example 3

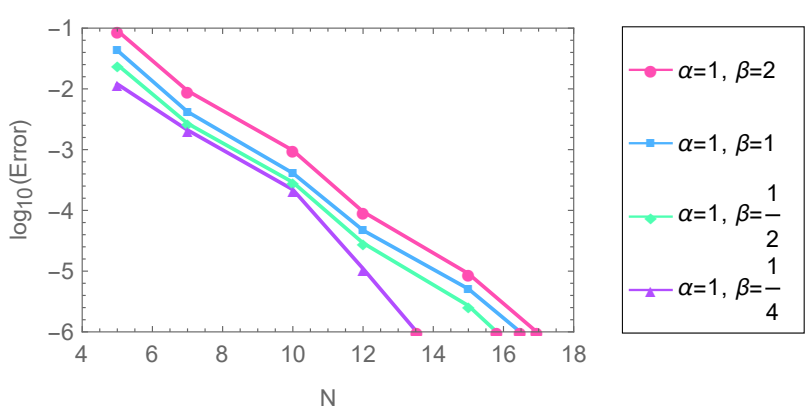

Fig. 6: Log-Error of Example 3

and the initial condition

$$
u(x, 0)=-\sin (\pi x),
$$

with the exact smooth solution

$$
u(x, t)=-e^{-\pi^{2} \mu t} \sin (\pi x) .
$$

We write

$$
u(x, t) \approx u^{N}(x, t)=\sum_{i=0}^{N} \sum_{j=0}^{N} c_{i j} T_{i}(x) L_{j}^{(\alpha, \beta)}(t),
$$

where $N$ is even number and $T_{i}(x)$ denotes the Chebyshev polynomials of degree $i$. The residual of Eq. (45) is given by

$$
\begin{aligned}
R(x, t)= & u_{t}^{N}(x, t)-\mu u_{x x}^{N}(x, t)+u^{N}(x, t) u_{x}^{N}(x, t) \\
& -\frac{\pi}{2} e^{-2 \pi^{2} \mu t} \sin (2 \pi x),
\end{aligned}
$$

we select the following collocation points

$x_{i}=\cos \left(\frac{(2 i+1) \pi}{2 N+2}\right), \quad t_{j}=\frac{j \tau}{N+1}, \quad 0 \leq i, j \leq N+1$,

where $x_{i}$ are the distinct roots of the first kind Chebyshev polynomial $T_{N+1}(x)$ and $t_{j}$ are the equidistant Riemann 
points. Now we have the following system of nonlinear algebraic equations

$$
\begin{aligned}
R\left(x_{i}, t_{j}\right) & =0, \quad 0 \leq i, j \leq N-1 \\
u\left( \pm 1, t_{j}\right) & =0, \quad 0 \leq j \leq \frac{N}{2}-1 \\
u\left(x_{i}, 0\right) & =-\sin \left(\pi x_{i}\right), \quad 0 \leq i \leq N
\end{aligned}
$$

The dimension of this system is $(N+1)^{2}$, thanks to the Newton's iterative method for solving this nonlinear system and finally we obtain the solution. We solve this system for the case corresponding to $\mu=0.02, \tau=10, N=20$ and $(\alpha, \beta)=\left(\frac{3}{2}, \frac{1}{2}\right)$. In Figures 7,8 , we depict the approximate solution of Eq. (45) and the error $E=u-u^{N}$, respectively.

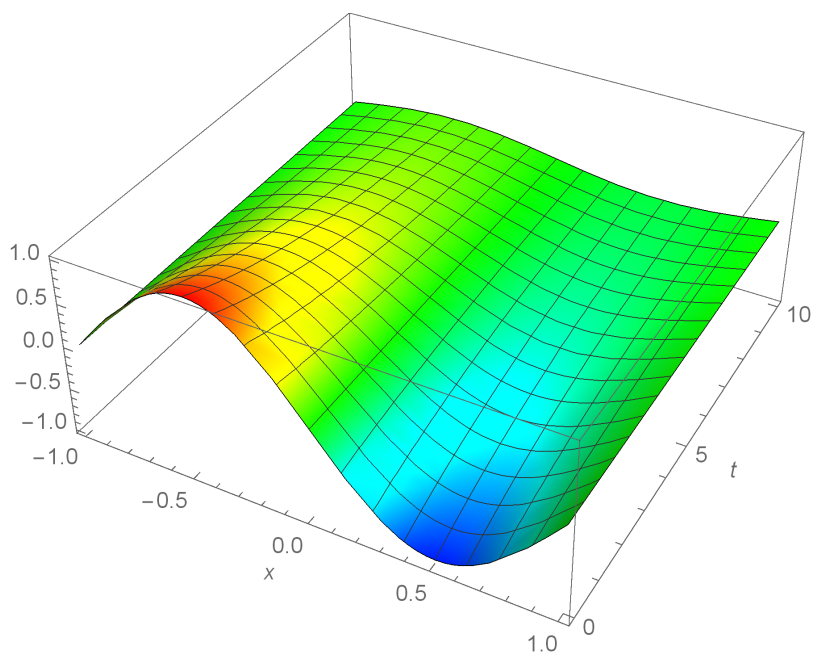

Fig. 7: Approximate solution of Example 4

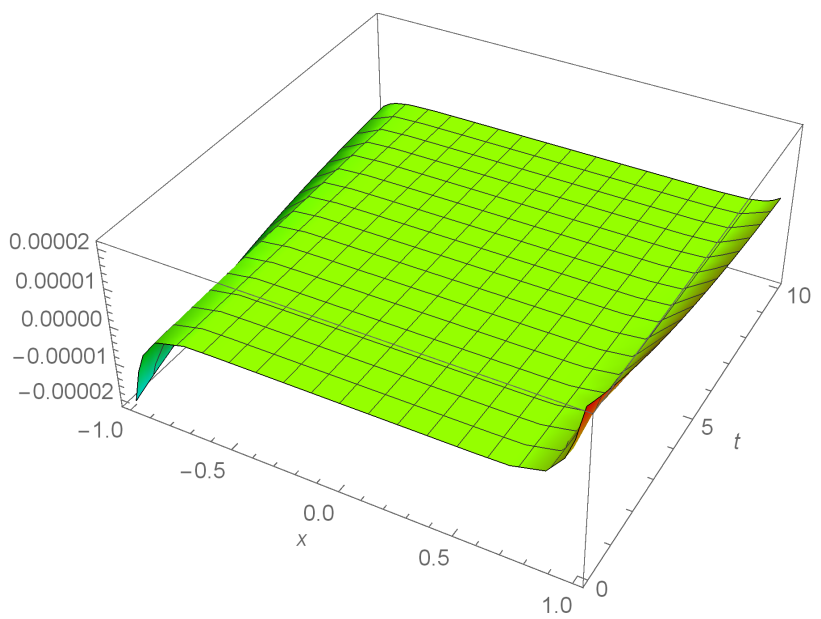

Fig. 8: Error of Example 4

\section{Concluding Remarks}

A formula expressing the modified generalized Laguerre coefficients of a general-order derivative of an infinitely differentiable function in terms of its original coefficients is proved, and a formula expressing explicitly the derivatives of modified Laguerre polynomials of any degree and for any order as a linear combination of suitable modified Laguerre polynomials is deduced. A formula for the modified Laguerre coefficients of the moments of one single modified Laguerre polynomial of certain degree is given. Four applications were implemented to illustrate the effect of the new parameter $\beta$ on the convergence and accuracy of the methods.

\section{References}

[1] S. Odake, R. Sasaki. Infinitely many shape-invariant potentials and cubic identities of the Laguerre and Jacobi polynomials. Journal of Mathematical Physics, 51(5):053513, 2010.

[2] J.S. Dehesa, R.J. Yáñez, A.I. Aptekarev, V. Buyarov. Strong asymptotics of Laguerre polynomials and information entropies of two-dimensional harmonic oscillator and onedimensional coulomb potentials. Journal of Mathematical Physics, 39(6):3050-3060, 1998.

[3] T. Tanaka. N-fold supersymmetry and quasi-solvability associated with $\times 2$-Laguerre polynomials. Journal of Mathematical Physics, 51(3):032101, 2010.

[4] R. Sasaki, S. Tsujimoto, A. Zhedanov. Exceptional Laguerre and Jacobi polynomials and the corresponding potentials through darboux-crum transformations. Journal of Physics A: Mathematical and Theoretical, 43(31):315204, 2010.

[5] G. Szego. Orthogonal polynomials, volume 23. American Mathematical Soc., 1939.

[6] R. Garrappa, M. Popolizio. On the use of matrix functions for fractional partial differential equations. Mathematics and Computers in Simulation, 81(5):1045-1056, 2011.

[7] B.J. Noye, M. Dehghan. New explicit finite difference schemes for two-dimensional diffusion subject to specification of mass. Numerical Methods for Partial Differential Equations, 15(4):521-534, 1999.

[8] Y. Zhao, W. Bu, X. Zhao, Y. Tang. Galerkin finite element method for two-dimensional space and time fractional blochtorrey equation. Journal of Computational Physics, 350:117135, 2017.

[9] W. Bu, Y. Tang, Y. Wu, J. Yang. Finite difference/finite element method for two-dimensional space and time fractional blochtorrey equations. Journal of Computational Physics, 293:264279, 2015.

[10] M.A. Zaky, E.H. Doha, T.M. Taha, D. Baleanu. New recursive approximations for variable-order fractional operators with applications. Mathematical Modelling and Analysis, 23:227239, 2018. 
[11] S. Chandrasekhar, S. Chandrasekhar. An introduction to the study of stellar structure, volume 2. Courier Corporation, 1957.

[12] H.T. Davis. Introduction to nonlinear differential and integral equations. Courier Corporation, 1962.

[13] W.M. Abd-Elhameed. New Galerkin operational matrix of derivatives for solving Lane-Emden singular-type equations. The European Physical Journal Plus, 130(3):52, 2015.

[14] R. Gupta, A.S. Mujumdar. Aerodynamics of a vibrated fluid bed. The Canadian Journal of Chemical Engineering, 58(3):332-338, 1980.

[15] J.P. Boyd. An analytical and numerical study of the twodimensional bratu equation. Journal of Scientific Computing, 1(2):183-206, 1986.

[16] M. Lemou, P.H. Chavanis. Escape of stars from gravitational clusters in the chandrasekhar model. Physica A: Statistical Mechanics and its Applications, 389(5):1021-1040, 2010.

[17] H. Bateman. Some recent researches on the motion of fluids. Monthly Weather Review, 43(4):163-170, 1915.

[18] J.M. Burgers. Mathematical examples illustrating relations occurring in the theory of turbulent fluid motion. In Selected Papers of JM Burgers, pages 281-334. Springer, 1995.

[19] C.I. Gheorghiu. The Laguerre collocation method. In Spectral Methods for Non-Standard Eigenvalue Problems, pages 85110. Springer, 2014.

[20] K. Parand, A. Taghavi. Rational scaled generalized Laguerre function collocation method for solving the blasius equation. Journal of Computational and Applied Mathematics, 233(4):980-989, 2009.

[21] J. Shen. Stable and efficient spectral methods in unbounded domains using Laguerre functions. SIAM Journal on Numerical Analysis, 38(4):1113-1133, 2000.

[22] O. Coulaud, D. Funaro, O. Kavian. Laguerre spectral approximation of elliptic problems in exterior domains. Computer Methods in Applied Mechanics and Engineering, 80(1-3):451458, 1990.

[23] A.H. Bhrawy, M.A. Zaky. An improved collocation method for multi-dimensional space-time variable-order fractional Schrödinger equations. Applied Numerical Mathematics, 111:451-458, 197-218, 2017.

[24] M.A. Zaky. An improved tau method for the multi-dimensional fractional Rayleigh-Stokes problem for a heated generalized second grade fluid. Computers \& Mathematics with Applications, 2017.

[25] B.Y. Guo, J. Shen, C.L. Xu. Generalized Laguerre approximation and its applications to exterior problems. Journal of computational Mathematics, pages 113-130, 2005.

[26] T.J. Wang, B.Y. Guo. Composite generalized LaguerreLegendre pseudospectral method for fokker-planck equation in an infinite channel. Applied Numerical Mathematics, 58(10):1448-1466, 2008.

[27] A. Rahmoune. Spectral collocation method for solving fredholm integral equations on the half-line. Applied Mathematics and Computation, 219(17):9254-9260, 2013.

[28] A.H. Bhrawy, A.A. Al-Zahrani, Y.A. Alhamed, D. Baleanu. A new generalized Laguerre-gauss collocation scheme for numerical solution of generalized fractional pantograph equations. Rom. J. Phys, 59(7-8):646-657, 2014.

[29] A. Karageorghis. A note on the chebyshev coefficients of the moments of the general order derivative of an infinitely differentiable function. Journal of Computational and Applied
Mathematics, 21(3):383-386, 1988.

[30] T.N. Phillips. On the Legendre coefficients of a general-order derivative of an infinitely differentiable function. IMA Journal of Numerical Analysis, 8(4):455-459, 1988.

[31] E.H. Doha. The coefficients of differentiated expansions and derivatives of ultraspherical polynomials. Computers \& Mathematics with Applications, 21(2):115-122, 1991.

[32] E.H. Doha. On the coefficients of differentiated expansions and derivatives of Jacobi polynomials. Journal of Physics A: Mathematical and General, 35(15):3467, 2002.

[33] E.H. Doha. On the connection coefficients and recurrence relations arising from expansions in series of hermite polynomials. Integral Transforms and Special Functions, 15(1):13-29, 2004.

[34] E.H. Doha and WM Abd-Elhameed. On the coefficients of integrated expansions and integrals of chebyshev polynomials of third and fourth kinds. Bull. Malays. Math. Sci. Soc.(2), 37(2):383-398, 2014.

[35] E.H. Doha. The first and second kind chebyshev coefficients of the moments for the general order derivative on an infinitely differentiable function. International journal of computer mathematics, 51(1-2):21-35, 1994.

[36] E.H. Doha, S.I. El-Soubhy. On the Legendre coefficients of the moments of the general order derivative of an infinitely differentiable function. International journal of computer mathematics, 56(1-2):107-122, 1995.

[37] E.H. Doha. The ultraspherical coefficients of the moments of a general-order derivative of an infinitely differentiable function. Journal of computational and applied mathematics, 89(1):5372, 1998.

[38] E.H. Doha. On the connection coefficients and recurrence relations arising from expansions in series of Laguerre polynomials. Journal of Physics A: Mathematical and General, 36(20):5449, 2003.

[39] W.M. Abd-Elhameed. New Connection Formulae Between (p,q)Fibonacci Polynomials and Certain Jacobi Polynomials. Journal of Inequalities and Special Functions, 7(2):1-16, 2016.

[40] W.M. Abd-Elhameed. New formulae between Jacobi polynomials and some fractional Jacobi functions generalizing some connection formulae. Analysis and Mathematical Physics, http://doi.org/10.1007/s13324-017-0183-7.

[41] W.M. Abd-Elhameed. New formulae of squares of some Jacobi polynomials via hypergeometric functions. Hacettepe Journal of Mathematics and Statistics, 46(2):165-176, 2017.

[42] V.G. Bagrov, D. Gitman. Exact solutions of relativistic wave equations, volume 39. Springer Science \& Business Media, 1990.

[43] A.F. Nikiforov, V.B. Uvarov. Special functions of mathematical physics, volume 205. Springer, 1988.

[44] D. Gottlieb, S.A. Orszag. Numerical analysis of spectral methods: theory and applications. SIAM, 1977.

[45] S. Lewanowicz. Properties of the polynomials associated with the Jacobi polynomials. mathematics of computation, 47(176):669-682, 1986.

[46] A. Abramowitz, I.A. Stegun. Handbook of Mathematical Functions: With Formulas, Graphs, and Mathematical Tables. Dover Publications, 1970. 